\title{
Reconfiguration of Resonance Characteristics for Terahertz U-Shape Metamaterial Using MEMS Mechanism
}

\author{
Yu-Sheng Lin, Chia-Yi Huang, and Chengkuo Lee, Member, IEEE
}

\begin{abstract}
We present the design, simulation, fabrication, and characterization of an out-of-plane reconfiguration of terahertz (THz) U-shape metamaterial. The U-shape metamaterial is consisted of bilayer cantilevers with different coefficient of thermal expansion. The electromagnetic tunability of U-shape metamaterial is accomplished by using electrostatic actuation mechanism to provide higher tuning range at lower driving voltage. The bilayer cantilevers are actuated toward the substrate by gradually increasing the bias and, then, completely snapped down when the bias reached the critical pull-in voltage. Therefore, this device can control the resonant frequency actively. The experimental results indicate that the device possesses $0.51-\mathrm{THz}$ tuning range with polarization dependence compared to de bias of 0 and $12 \mathrm{~V}$. Moreover, this device can be a THz switch when rotated to different angle with respect to the polarization of incident light. Hence, such adaptive metamaterial device offers significant potential in realizing the multifunctionality in optical filter, polarization controller, and optical switch applications.
\end{abstract}

Index Terms-Microelectromechanical systems (MEMS), electromagnetic propagation, tunable filters, optical switches.

\section{INTRODUCTION}

$\mathbf{M}$ ETAMATERIALS are artificial composites which exhibit strong electric and magnetic responses to manipulate the amplitude, direction, polarization, wavelength and phase of electromagnetic waves. The unique characteristics of electromagnetic metamaterial make diversified applications become true where these applications include cloaking devices, high-resolution superlenses, perfect absorber, security screening, medical imaging, and non-destructive testing [1]-[5], etc. Research has been actively carried out in this field to characterize how these extreme electromagnetic properties achieved through the interaction of the incident electromagnetic wave with shape and size of designed metamaterial patterns. One of the classic metamaterial structures is considered as a ring with a common split. This kind of metamaterial structure is named as split-ring resonator (SRR). An inductive effect is attributed to a current induced in the SRR ring, i.e., flowing around the

Manuscript received June 17, 2014; revised September 3, 2014 and September 16, 2014; accepted October 4, 2014. This work was supported by MOE2012T2-2-154 (Monolithic Integrated Si/AIN Nanophotonics Platform for Optical NEMS and OEICs) under WBS No. R-263-000-A59-112.

Y.-S. Lin and C. Lee are with the Department of Electrical and Computer Engineering, National University of Singapore, Singapore 117581 (e-mail: elelinys@nus.edu.sg; elelc@nus.edu.sg).

C.-Y. Huang is with the Department of Applied Physics, Tunghai University, Taichung 40704, Taiwan (e-mail: chiayihuang@thu.edu.tw).

Color versions of one or more of the figures in this paper are available online at http://ieeexplore.iee.org.

Digital Object Identifier 10.1109/JSTQE.2014.2361840
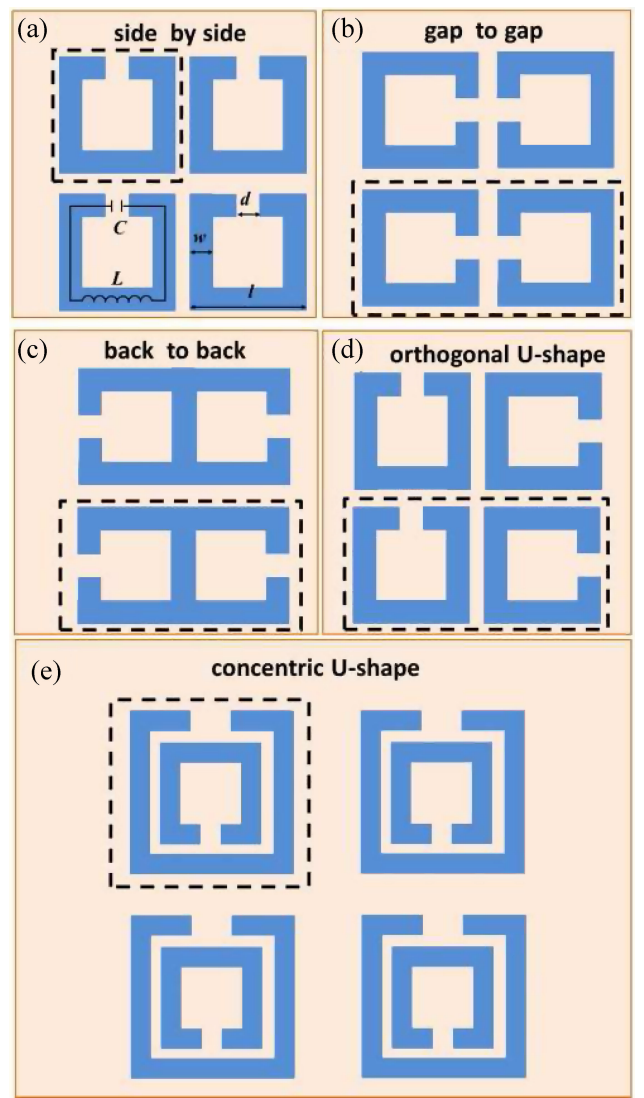

Fig. 1. Five classical configurations of SRR. (a) Side by side of U-shape, (b) gap to gap of U-shape, (c) back to back of U-shape, (d) orthogonal U-shape, and (e) concentric U-shape, respectively. The black dash square indicates the unit cell of each configuration.

ring, as well as a capacitive effect across the split. Therefore, the SRR can be described qualitatively in terms of its equivalent circuit. Among various SRR designs, there are five typical U-shape SRR configurations such as side by side [see Fig. 1(a)] [6]-[11], gap to gap [see Fig. 1(b)] [12]-[16], back to back [see Fig. 1(c)] [3], [17], [18], orthogonal U-shape [see Fig. 1(d)] [19], [20], and concentric U-shape [see Fig. 1(e)] [21]-[24] as summarized in Fig. 1. Each of U-shape SRR comprises a capacitor-like structure which is coupled to the electric field and inductance is provided in the parallel-connected circuit with the resonant frequency of $\omega=\sqrt{1 / L C}$ as shown in Fig. 1(a). Here, $C=\varepsilon_{0} \varepsilon_{c} w t / d$ and $L=\mu_{0} l^{2} / t$ refer to the respective capacitance and inductance of SRR, where $w$ is the width of SRR, $d$ is the width of capacitor split, $t$ is the metal thickness, $l$ is 
the size of the SRR, $\varepsilon_{0}$ is the free space permittivity, and $\varepsilon_{c}$ is the relative permittivity of the materials in the capacitor split, respectively [8].

Recently, the electromagnetic tunability of metamaterial has attracted intense research interest, since the electromagnetic response of the metamaterial can be actively controlled through external stimulus, which is of great significance in real time applications [24]-[26], [28], [29]. Such active control of metamaterial characteristics is crucial in order to provide a flexible and versatile platform for mimicking fundamental physical effects. To realize the electromagnetic tunability, various approaches have been demonstrated based on the U-shape patterns to increase the flexibility in applications, such as changing the effective electromagnetic properties via dielectric layers [16], [21], semiconductor diodes [7], thermal control [9], [17], [24], liquid crystals [11], laser light illuminating [10], [14], [19] and magnetic field [23], etc. Alternatively, microelectromechanical systems (MEMS) based techniques are well developed for the realization of movable microstructures. The structural reconfiguration is a straightforward way to control the electromagnetic properties including amplitude, polarization, and directionality for the metamaterial structures. The metamaterial properties can be directly modified by reconfiguring the unit cell which is the fundamental building block of metamaterials. Among these MEMS approaches, electrostatic comb drive actuator has been utilized to change the in-plane spacing of metamaterial unit cell [15], [30]-[32], while surface-micromachined out-of-place stress beams in each metamaterial unit cell can be deformed toward the substrate due to the attractive force from a parallelplate electrostatic actuator [33]-[38]. On the other hand, the electrothermal actuation mechanisms have been reported as well [27], [39]. Therefore, MEMS-based metamaterials are not only promising in tunability but also good at widespread applications [30]-[39].

To increase the tuning range of metamaterial resonance, our previous report [34] has demonstrated the concentric U-shape metamaterial using out-of-plane actuation [see Fig. 1(e)] with larger tuning range $(0.50 \mathrm{THz})$ than above-mentioned data. However, there is no literature demonstrated the SRR offering multi-functionality in optical filter, polarization controller, and optical switch simultaneously. In this study, we explore and demonstrate a reconfiguration of U-shape metamaterial with a comparable tuning range to reference [34] by using an out-of-plane MEMS mechanism. Herein, the proposed Ushape metamaterial design is demonstrated to possess the multifunctionality simultaneously. This design exhibits not only increasing the tuning range of resonant frequency, but also having the polarization dependence property. Furthermore, this device can be used as an optical switch when the device is rotated to different angle with respect to the TE polarized incidence, hence allowing this device to be effectively used for optical switches and filters applications.

\section{Design of U-Shape Metamaterial}

The most effective way of adjusting metamaterial unit cell is making MEMS actuation structures as the U-shape metamaterial

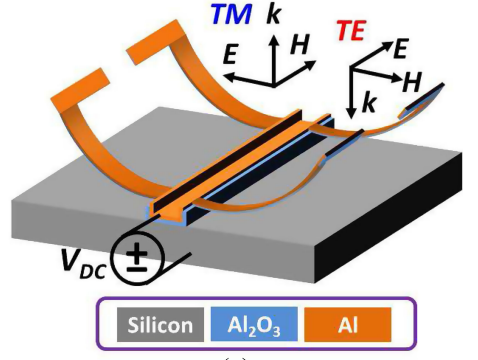

(a)

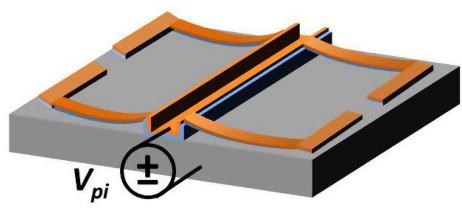

(b)

Fig. 2. Schematic drawings of an actively controlled U-shape metamaterial (a) without dc bias and (b) at pull-in voltage, respectively.

itself. Fig. 2(a) shows the schematic drawing of the proposed U-shape metamaterial unit cell without driving voltage, where the U-shape metamaterial unit cell has four bilayer cantilevers. The composition of each bilayer cantilever is $\mathrm{Al} / \mathrm{Al}_{2} \mathrm{O}_{3} / \mathrm{SiO}_{2}$ multilayers with 500/20/100-nm thickness on an n-type silicon wafer. The $\mathrm{SiO}_{2}$ layer was patterned as anchors for four bilayer cantilevers. In order to create the upward-bending structures, vaporized hydrofluoric acid was used to etch $\mathrm{SiO}_{2}$ layer and then release the bilayer cantilevers so as to create out-ofplane bending cantilevers for U-shape metamaterial unit cell because of residual stress owing to the different coefficient of thermal expansion (CTE) of $\mathrm{Al}$ and $\mathrm{Al}_{2} \mathrm{O}_{3}$ films. The detailed fabrication process flow can be referred to our previous results [34], [35]. The electromagnetic tunability of metamaterial device is achieved by changing the air gap between the flexible bilayer cantilevers of metamaterial and the substrate. This gap is changed due to the electrostatic force by applying a dc voltage on the bilayer cantilevers and the substrate. The series capacitance of the U-shape metamaterial device depends on the position or bending degree of the bilayer cantilevers. Regarding an intermediate state, the deformation of the bilayer cantilevers is determined by the balance between the electrostatic force and the restoring force of cantilever. When the dc voltage between the cantilevers and the substrate exceeds the critical pull-in voltage $\left(V_{p i}\right)$, the cantilevers become unstable and will be pulling down to the substrate as shown in Fig. 2(b). Hence, the electrostatic tunability of U-shape metamaterial device can be actively and continuously controlled via a driving voltage less than $V_{p i}$. Here, the geometry parameters of U-shape metamaterial unit cell are shown in Fig. 3(a). The unit cell is a square with an edge length of $104 \mu \mathrm{m}$. An inductive effect is introduced when the current induced in the connected U-shape flows around as well as a capacitive effect across the split. Therefore, the U-shape metamaterial device can be described qualitatively in terms of an equivalent circuit of a capacitor-like structure coupled to the electric field and connected in parallel providing inductance as shown in Fig. 3(b). Fig. 3(c) shows the SEM image of the 


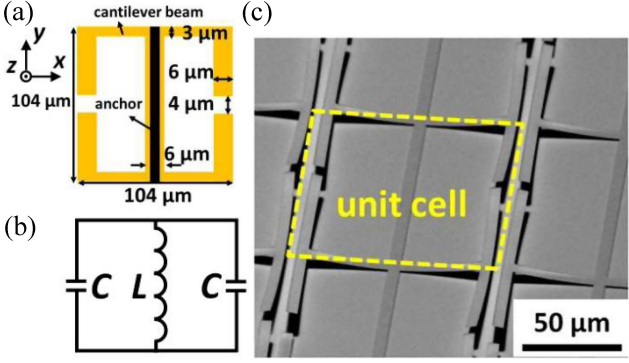

Fig. 3. (a) Layout of U-shape metamaterial unit cell and corresponding geometry parameters. (b) The equivalent circuit of U-shape metamaterial. (c) SEM image of U-shape metamaterial after releasing microstructures.

TABLE I

MATERIAL PROPERTIES

\begin{tabular}{lccccc}
\hline \hline Materials & $\begin{array}{c}\text { Young's } \\
\text { Modulus } \\
(\mathrm{GPa})\end{array}$ & $\begin{array}{c}\text { Poisson's } \\
\text { Ratio }\end{array}$ & $\begin{array}{c}\mathrm{CTE} \\
\left(\times 10^{-6} K^{-1}\right)\end{array}$ & $\begin{array}{c}\text { Electrical } \\
\text { Conductivity } \\
\left(\times 10^{6} \mathrm{~S} \cdot\right. \\
\left.\mathrm{m}^{-1}\right)\end{array}$ & $\begin{array}{c}\text { Density } \\
\left(\mathrm{g} \cdot \mathrm{cm}^{-3}\right)\end{array}$ \\
\hline Silicon & 169 & 0.30 & 2.568 & 0.025 & 2.33 \\
$\mathrm{Al}_{2} \mathrm{O}_{3}$ & 530 & 0.30 & 8.10 & - & 3.95 \\
$\mathrm{Al}$ & 70 & 0.30 & 23.1 & 35.5 & 2.70 \\
\hline \hline
\end{tabular}

U-shape metamaterial array after the bilayer cantilevers were released, the curve was calculated with an initial height of $15 \mu \mathrm{m}$. This gap between the metamaterial and substrate is an important tuning parameter, since different configurations of the unit cell can be obtained by simply adjusting the value of initial gap.

\section{SimUlation AND EXPERIMENT RESUlts OF U-SHAPE METAMATERIAL}

The correlation between the carrier excitations and incident electromagnetic waves of proposed U-shape metamaterial unit cell structure was used a commercial finite-difference time domain solver, CST Microwave Studio. The finite element simulations were performed using the material parameters listed in Table I. The program simulates a single unit cell shown in Fig. 3(a) with appropriate boundary conditions, i.e., perfect electric boundary condition in the $y z$ plane and perfect magnetic boundary condition in the $x z$ plane. Fig. 4 shows the simulation results of U-shape metamaterial at TE and TM polarized incidence, respectively, and with cantilevers under different bending states owing to different driving voltages. The TE polarization state represents the case in which the electric field is perpendicular to the split side of the ring, while TM occurs as the electric field is parallel to the split side. When we gradually shrink down the gap between U-shape metamaterial and substrate, the resonances are shifted. At TE polarized incidence, there are two resonant frequencies as shown in Fig. 4(a). The lower resonance is the inductive-capacitive $(L C)$ resonance, where oscillating charges generate circulating currents throughout the entire structure and results in a pure electric response. The surface current and electric field distributions are shown in Fig. 5(a) and (c), respectively. There is no significant electric field distribution

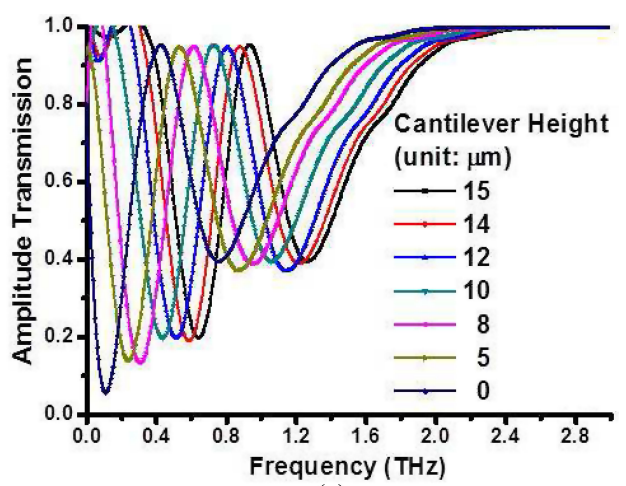

(a)

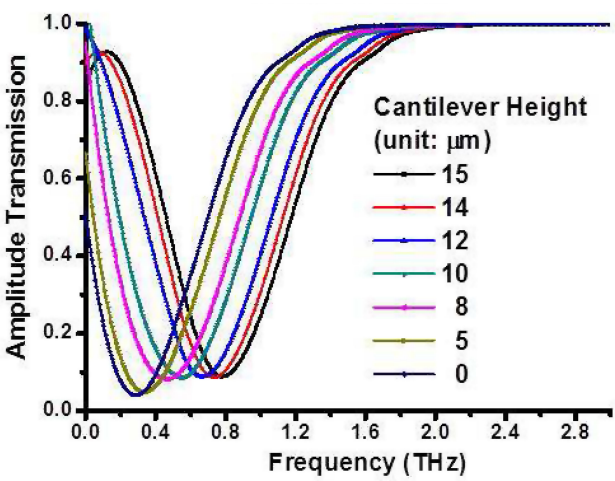

(b)

Fig. 4. Simulation results of U-shape metamaterial with different cantilever height at (a) TE and (b) TM polarized incidence, respectively.

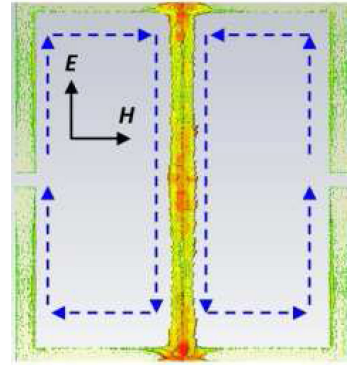

(a)

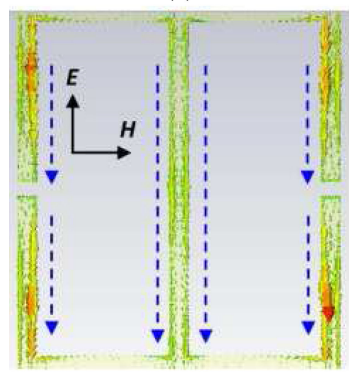

(c)

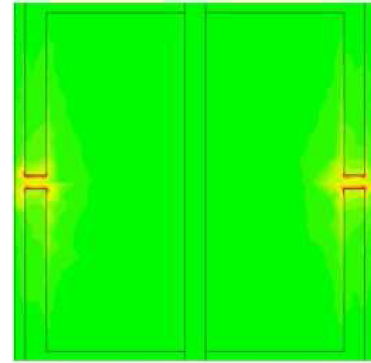

(b)

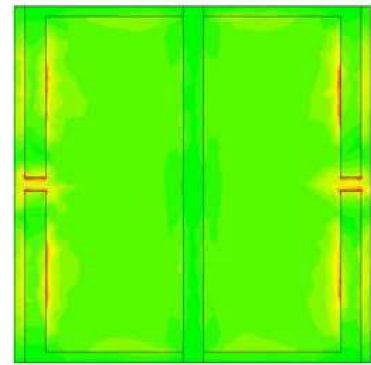

(d)
Fig. 5. Numerical simulation results of U-shape metamaterial at TE mode (a) and (b) are the surface current densities at the lower and second resonances, respectively, where the blue arrows denote the surface current directions. (c) and (d) are the corresponding electric field distributions of (a) and (b), respectively.

along the connecting metal lines between U-shape metamaterial at the fundamental resonant frequency. It indicates that the $\mathrm{U}$-shape metamaterial operates in the $L C$ resonance. This resonance shifts from 0.64 to $0.11 \mathrm{THz}$ in comparison with the initial state (cantilevers at an initial height of $15 \mu \mathrm{m}$ ) to the snap-down 


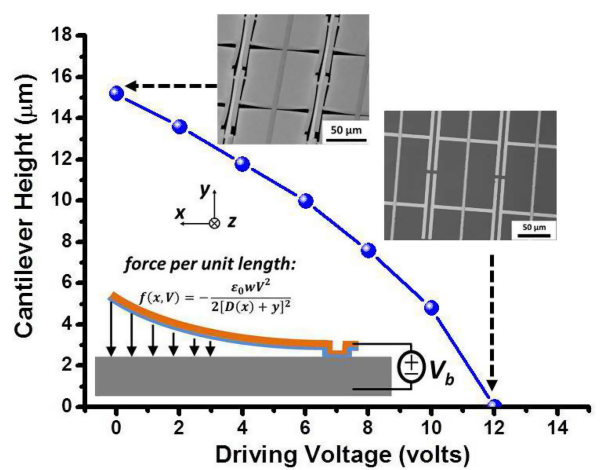

Fig. 6. (a) Driving voltages versus the displacement of U-shape metamaterial and corresponding SEM images of U-shape metamaterial without dc bias and at pull-in voltage. Left-bottom inset shows the schematic drawing of cantilever profile of $\mathrm{U}$-shape metamaterial after releasing and under driving voltage.

state. The second resonance is the dipole type electric resonance caused by in-phase dipolar current along the U-shape metamaterial sides or components parallel to the incident electric field. The surface current distribution is shown in Fig. 5(b) and the corresponding electric field distribution is shown in Fig. 5(d). The resonance shifts from 1.27 (initial state) to $0.75 \mathrm{THz}$ (snapdown state). At TM polarized incidence, because the electric field is oriented along a mirror plane for the U-shape structures, the resonance is purely attributed to the electrical response. The resonance is shifted from 0.78 (initial state) to $0.27 \mathrm{THz}$ (snapdown state) as shown in Fig. 4(b).

The electromechanical design of U-shape metamaterial is used stress-induced cantilever to form an out-of-plane U-shape beams for the electrostatic tunability. We used an external dc power supply to actuate the U-shape metamaterial cantilevers. The relationship of driving voltage and cantilever height is plotted in Fig. 6. Due to the CTE of two materials mismatch, the strain developed in the two materials must be equal at the boundary, which leads to [40], [41]

$$
\alpha_{1}(\Delta T)+\frac{P}{E_{1}\left(w t_{1}\right)}+\frac{t_{1}}{2 \rho}=\alpha_{2}(\Delta T)-\frac{P}{E_{2}\left(w t_{2}\right)}-\frac{t_{2}}{2 \rho}
$$

where subscripts 1 and 2 refer to parameters for $\mathrm{Al}$ and $\mathrm{Al}_{2} \mathrm{O}_{3}$ layers of the composite of cantilever, respectively. $E$ is Young's modulus, $\alpha$ is CTE of material, $\Delta T$ is the change of temperature, $w$ is the width of cantilever, $t$ is layer thickness of material, respectively. Thus, the change in radius of curvature is expressed as [40]

$$
\frac{1}{\rho}-\frac{1}{\rho_{0}}=\frac{2 \Delta \alpha \Delta T}{t_{1} C}
$$

where

$$
C=\frac{\left(1+\frac{E_{2} I_{2}}{E_{1} I_{1}}\right)\left(1+\frac{E_{2} t_{2}}{E_{1} t_{1}}\right)}{6 \frac{E_{2}}{E_{1}} \frac{t_{2}}{t_{1}}\left(1+\frac{t_{2}}{t_{1}}\right)}+\left(1+\frac{t_{2}}{t_{1}}\right) .
$$

The initial deflection of the cantilever beam along the length, $D(x)$ is given by

$$
D(x)=\frac{x^{2}}{2}\left(\frac{1}{\rho}-\frac{1}{\rho_{0}}\right) .
$$

Therefore, the initial deflection of the cantilever can be modeled if the geometry parameters and materials properties of two layers are known. The electrostatic force acting on the cantilever is given by $F=\left(\varepsilon A V^{2}\right) / 2 g^{2}$, where $\varepsilon$ is the permittivity of the medium, $A$ is the area of the electrodes, $V$ is the driving voltage, $g$ is the gap distance between the cantilever and substrate. This force equation equals to the sum of initial gap distance $D(x)$ and displacement $y$. We consider the force per unit length along the cantilever for a given bias voltage:

$$
E I \frac{d^{4} y}{d x^{4}}=f(x, V)=-\frac{\varepsilon_{0} w V^{2}}{2[D(x)+y]^{2}}
$$

where $f(x, V)$ denotes the static electrostatic force per unit cantilever length as a function of the position $x$ and the driving voltage $V . \varepsilon_{0}$ is the permittivity of free space. The electrostatic force per unit length along the cantilever is inversely proportional to the square of gap between metamaterial and substrate. The capacitance between the metamaterial and substrate can be expressed as

$$
C(x)=\int_{0}^{L} \frac{\varepsilon_{0} w d x}{\left[D(x)+(1 / k) t_{2}\right]}
$$

where $L$ is cantilever length and $k$ is the relative permittivity of $\mathrm{Al}_{2} \mathrm{O}_{3}$ layer. This stress-induced cantilever exhibits a very small gap at the joint of cantilever and substrate, i.e. the thickness of sacrificial layer, $\mathrm{SiO}_{2}$, is $100 \mathrm{~nm}$ thick in this study. The strong electrostatic force occurred at this small gap due to the applied dc bias will firstly pull down the portion of cantilever nearby the joint. Then the adjacent gap becomes small as well and the portion of cantilever on top of this gap will be pulled down to substrate. Gradually more portion of cantilever is at down state. Hence, the above calculations can be assumed the neglecting fringing electric field. The final balanced position and shape of cantilever is subject to the balance between electrostatic force and cantilever restoring force. By leveraging this unique curved cantilever structure, the driving voltage is reduced. This design significantly increase the moving range of the SRR structures compared with the counterparts using in-plane movable MEMS structures. The cantilever displacement of the deformed cantilever is the most important factor for tuning the equivalent capacitance of U-shape metamaterial device, and consequently the resonance frequency of the resonators. Hence it is necessary to study the relationship between the cantilever profile and driving voltages. During the operation, the cantilever profile is proportional to the driving voltage by using equation (5). The U-shape metamaterial cantilevers starts from height of $15 \mu \mathrm{m}$ and deforms gradually to the substrate by increasing the driving voltage. Ultimately, the cantilevers forced and snapped down to substrate, which initial state and snap-down state are shown in the inset SEM images of Fig. 6.

The electromagnetic response of U-shape metamaterial device was measured by using the transmission spectra using terahertz time domain spectroscopy (THz-TDS, TeraView TPS 3000) system in transmission mode. Under various actuation states, the $\mathrm{THz}$ signal was measured in the time domain after transmitting through the U-shape metamaterial device, which were transformed to frequency domain and normalized by 


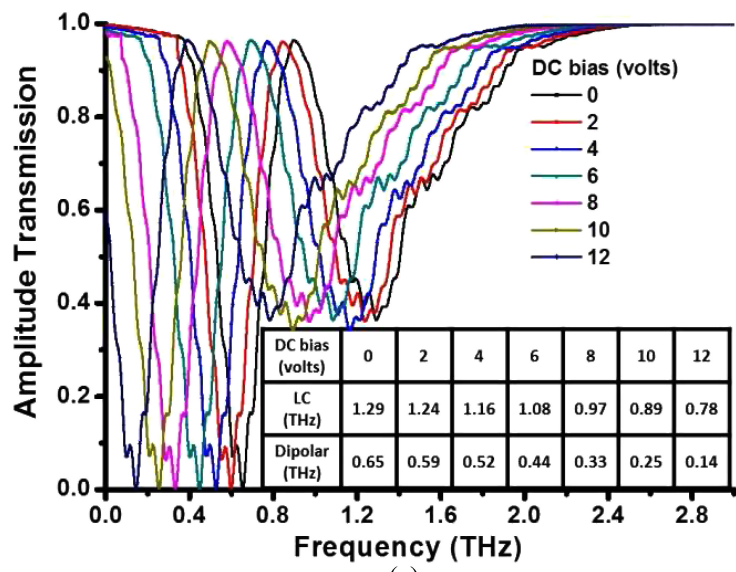

(a)

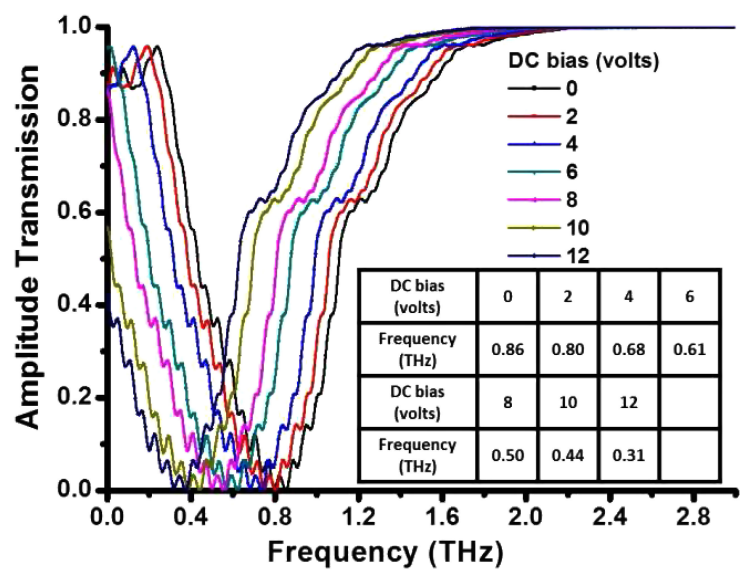

(b)

Fig. 7. Experimental results of U-shape metamaterial at different dc bias. (a) and (b) are U-shape metamaterial at TE and TM polarized incidence, respectively.

using a bare silicon substrate as the reference. All the measurements were done at room temperature and in a dry atmosphere to mitigate water vapor absorption. Fig. 7(a) and (b) show the measurement results of U-shape metamaterial device operating at TE and TM polarized incidence, respectively. The polarization of the incident wave is indicated in Fig. 2(a). The TE and TM polarization states represent the case in which the electric field is perpendicular and parallel to the split side of the Ushape, respectively. For TE polarized incidence, there are two distinct resonances in the spectrum as shown in Fig. 7(a). The resonant frequencies are shifted by $0.51 \mathrm{THz}$ for $L C$ and dipolar resonances, respectively in comparison to the device without driving voltage and it is snapped down to substrate at $12 \mathrm{~V} \mathrm{dc}$ bias. In Fig. 7(b), it is because the electric field is oriented along an entire U-shape structures, the resonance is purely due to the electrical response. By applying dc bias on device, the transmission dip is shifted from 0.86 to $0.31 \mathrm{THz}$ at $12 \mathrm{~V}$ dc bias, resulting in a tuning range of $0.55 \mathrm{THz}$. It evidently shows the $\mathrm{U}$-shape metamaterial device is polarization dependent for TE and TM polarized incidence, while a large tuning range of 0.51 and $0.55 \mathrm{THz}$ for TE and TM mode, respectively, compared the device without dc bias to snap-down state. For easy comparison, the measured resonant frequencies of U-shape metamaterial

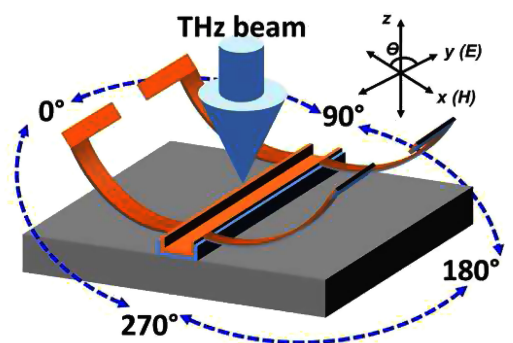

(a)

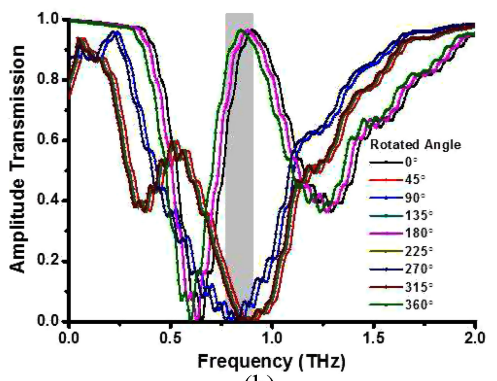

(b)

Fig. 8. (a) Schematic drawing of U-shape metamaterial with different rotated angle at TE polarized incident beam. (b) Experimental results of U-shape metamaterial measured by using THz-TDS system.

device are further summarized in the inset table of Fig. 7. These results are comparable with the simulation results of $\mathrm{TE}$ and TM polarized incidence in Fig. 4. The experimental results of U-shape metamaterial device indicate that the U-shape metamaterial is not only polarization-dependent but providing larger tuning range as well. It demonstrates the unique potential of out-of-plane movable metamaterials in filter with large tuning range for electromagnetic response.

\section{Characterizations OF U-Shape Metamaterial}

To characterize the polarization dependent characteristic of the U-shape metamaterial device, another chip is released and its transmission spectra were measured with no input dc bias using a THz-TDS system. The device was oriented vertically along the $z$-axis with a normal TE polarized incidence as shown in Fig. 8(a). The transmitted light was then measured along the $\mathrm{x}$ and $\mathrm{y}$ axes with respect to $\theta=0$ or $\theta=90$, respectively. In Fig. 8(b), the black curve shows the resonance frequency is 0.65 and $1.29 \mathrm{THz}$ for $L C$ and dipolar mode, respectively, when the device is not rotated. This result is identical with that of measurement at TE polarized incidence as shown in Fig. 7(a). At a rotation angle of $45^{\circ}$, the resonances are shifted to 0.39 and $0.91 \mathrm{THz}$ for $L C$ and dipolar mode, respectively. When continuously rotated the device to $90^{\circ}$ with respect to the $z$-axis in the $x y$ plane, the device loses the resonance of TE polarized incidence characteristic and approaches to the resonance at TM polarized incidence, where the resonance is $0.84 \mathrm{THz}$ comparable to the results of Fig. 7(b). In Fig. 8(b), the resonances of U-shape metamaterial device are similar at a rotation angle of $0^{\circ}, 180^{\circ}$, and $360^{\circ}$, that resonant behavior is comparable to the TE resonances in Fig. 7(a). While the device was rotated to $45^{\circ}$, $315^{\circ}, 225^{\circ}$, and $315^{\circ}$, those TE resonances loss gradually, and then transmission characteristic approaches to TM resonances 
with $90^{\circ}$ and $270^{\circ}$. Notably, the optical behavior of U-shape metamaterial has a switch function at a gray-shaded region in Fig. 8 (b) by rotating the device with $0^{\circ}$ to $90^{\circ}$, or $90^{\circ}$ to $180^{\circ}$, individually, i.e. the amplitude transmission is switched from $1\left(0^{\circ}\right)$ to $0\left(90^{\circ}\right)$ and $0\left(90^{\circ}\right)$ to $1\left(180^{\circ}\right)$ at $0.8-0.9 \mathrm{THz}$ frequency range.

\section{CONCLUSION}

In conclusion, a U-shape metamaterial using out-of-plane MEMS actuation mechanism is developed and characterized. Such an out-of-plane motion of cantilevers enables the control of resonant frequency by changing the gap between the bilayer cantilevers of metamaterial and the substrate. The experimental results show that the U-shape metamaterial device is a $\mathrm{THz}$ tunable filter and a polarization controller. The polarization dependent results of U-shape metamaterial demonstrate a tuning range of 0.51 and $0.55 \mathrm{THz}$ with respect to resonances of TE and TM mode, respectively. Furthermore, the U-shape metamaterial device can be an optical switch at $\mathrm{THz}$ frequency range when operated without dc bias and with a different rotation angle. This U-shape metamaterial device does not only promise in polarization control of light, but also creates inspirations in active switching and optical filters applications.

\section{REFERENCES}

[1] D. Shin, Y. Urzhumov, Y. Jung, G. Kang, S. Baek, M. Choi, H. Park, K. Kim, and D. R. Smith, "Broadband electromagnetic cloaking with smart metamaterials," Nature Commun., vol. 3, no. 1213, pp. 1-8, Nov. 2012.

[2] N. Fang and X. Zhang, "Imaging properties of a metamaterial superlens," Appl. Phys. Lett., vol. 82, no. 2, pp.161-163, Jan. 2003.

[3] N. I. Landy, S. Sajuyigbe, J. J. Mock, D. R. Smith, and W. J. Padilla, "Perfect metamaterial absorber," Phys. Rev. Lett., vol. 100, no. 20, p. 207402, May 2008.

[4] D. R. Smith, D. C. Vier, N. Kroll, and S. Schultz, "Direct calculation of permeability and permittivity for a left-handed metamaterial," Appl. Phys. Lett., vol. 77, no. 14, pp. 2246-2248, Oct. 2000.

[5] U. Leonhardt, "Optical conformal mapping," Science, vol. 312, no. 5781, pp. 1777-1780, Jun. 2006

[6] C. Rockstuhl, F. Lederer, C. Etrich, T. Zentgraf, J. Kuhl, and H. Giessen, "On the reinterpretation of resonances in split-ring-resonators at normal incidence," Opt. Exp., vol. 14, no. 19, pp. 8827-8836, Sep. 2006.

[7] S. Myoga, T. Amemiya, A. Ishikawa, N. Nishiyama, T. Tanaka, and S. Arai, "Carrier-concentration-dependent resonance frequency shift in a metamaterial loaded semiconductor," J. Opt. Soc. Amer. B, vol. 29, no. 8, pp. 2110-2115, Aug. 2012.

[8] S. Linden, C. Enkrich, M. Wegener, J. Zhou, T. Koschny, C. M. Soukoulis, "Magnetic response of metamaterials at 100 terahertz," Science, vol. 306, no. 5700, pp. 1351-1353, Nov. 2004.

[9] H. Tao, A. C. Strikwerda, K. Fan, W. J. Padilla, X. Zhang, and R. D. Averitt, "Reconfigurable terahertz metamaterials," Phys. Rev. Lett., vol. 103, no. 14, pp. 147401, Oct. 2009.

[10] W. J. Padilla, A. J. Taylor, C. Highstrete, M. Lee, and R. D. Averitt, "Dynamical electric and magnetic metamaterial response at terahertz frequencies," Phys. Rev. Lett., vol. 96, no. 10, p. 107401, Mar. 2006.

[11] Z. Liu, C. Y. Huang, H. Liu, X. Zhang, and C. Lee, "Resonance enhancement of terahertz metamaterials by liquid crystals/indium tin oxide interfaces," Opt. Exp., vol. 21, no. 21, pp. 6519-6525, Mar. 2013.

[12] C. Y. Chen, I. W. Un, N. H. Tai, and T. J. Yen, "Asymmetric coupling between subradiant and superradiant plasmonic resonances and its enhanced sensing performance," Opt. Exp., vol. 17, no. 17, pp. 15372-15380, Aug. 2009.

[13] T. Driscoll, H. T. Kim, B. G. Chae, B. J. Kim, Y. W. Lee, N. M. Jokerst, S. Palit, D. R. Smith, M. D. Ventra, and D. N. Basov, "Memory metamaterials," Science, vol. 325, no. 5947, pp. 1518-1521, Sep. 2009.
[14] J. Gu, R. Singh, X. Liu, X. Zhang, Y. Ma, S. Zhang, S. A. Maier, Z. Tian, A. K. Azad, H. T. Chen, A. J. Taylor, J. Han, and W. Zhang, "Active control of electromagnetically induced transparency analogue in terahertz metamaterials," Nature Commun., vol. 3, no. 1151, pp. 1-6, Nov. 2012

[15] W. M. Zhu, A. Q. Liu, X. M. Zhang, D. T. Tsai, T. Bourouina, J. H. Teng, X. H. Zhang, H. C. Guo, H. Tanoto, T. Mei, G. Q. Lo, and D. L. Kwong, "Switchable magnetic metamaterials using micromachining processes," Adv. Mater, vol. 23, no. 15, pp. 1792-1796, Feb. 2011.

[16] T. Driscoll, G. O. Andreev, D. N. Basov, S. Palit, S. Y. Cho, N. M. Jokerst, and D. R. Smith, "Tuned permeability in terahertz split-ring resonators for devices and sensors," Appl. Phys. Lett., vol. 91, no. 6, p. 062511, Sep. 2007.

[17] J. Zhu, J. Han, Z. Tian, J. Gu, Z. Chen, and W. Zhang, "Thermal broadband tunable terahertz metamaterials," Opt. Commun., vol. 284, no. 12, pp. 3129-3133, Mar. 2011.

[18] N. Kumar, A. C. Strikwerda, K. Fan, X. Zhang, R. D. Averitt, P. C. M. Planken, and A. J. L. Adam, "THz near-field Faraday imaging in hybrid metamaterials," Opt. Exp., vol. 20, no. 10, pp. 11277-11287, May 2012.

[19] D. R. Chowdhury, R. Singh, A. J. Taylor, H. T. Chen, and A. K. Azad, "Ultrafast manipulation of near field coupling between bright and dark modes in terahertz metamaterial," Appl. Phys. Lett., vol. 102, no. 1, pp. 011122-1-011122-5, Jan. 2013.

[20] N. Liu, S. Kaiser, and H. Giessen, "Magnetoinductive and electroinductive coupling in plasmonic metamaterial molecules," Adv. Mater., vol. 20, no. 23, pp. 4521-4525, Oct. 2008.

[21] T. J. Yen, W. J. Padilla, N. Fang, D. C. Vier, D. R. Smith, J. B. Pendry, D. N. Basov, and X. Zhang, "Terahertz magnetic response from artificial materials," Science, vol. 303, no. 5663, pp. 1494-1496, Mar. 2004.

[22] J. F. O'Hara, R. Singh, I. Brener, E. Smirnova, J. Han, A. J. Taylor, and W. Zhang, "Thin-film sensing with planar terahertz metamaterials: Sensitivity and limitations," Opt. Exp., vol. 16, no. 3, pp. 1786-1795, Feb. 2008.

[23] B. Jin, C. Zhang, S. Engelbrecht, A. Pimenov, J. Wu, Q. Xu, C. Cao, J. Chen, W. Xu, L. Kang, and P. Wu, "Low loss and magnetic fieldtunable superconducting terahertz metamaterial," Opt Exp., vol. 18, no. 18, pp. 17504-17509, Aug. 2010.

[24] R. Singh, Z. Tian, J. Han, C. Rockstuhl, J. Gu, and W. Zhang, "Cryogenic temperatures as a path toward high-Q terahertz metamaterials," Appl. Phys. Lett., vol. 96, no. 7, p. 071114, Feb. 2010.

[25] H. T. Chen, W. J. Padilla, J. M. O. Zide, A. C. Gossard, A. J. Taylor, and R. D. Averitt, "Active terahertz metamaterial devices," Nature, vol. 444 no. 7119, pp. 597-600, Nov. 2006

[26] H. T. Chen, W. J. Padilla, M. J. Cich, A. K. Azad, R. D. Averitt, and A. J. Taylor, "A metamaterial solid-state terahertz phase modulator," $\mathrm{Na}$ ture Photonics, vol. 3, no. 3, pp. 148-151, Mar. 2009.

[27] J. Y. Ou, E. Plum, L. Jiang, and N. I. Zheludev, "Reconfigurable photonic metamaterials," Nano Lett., vol. 11, no. 5, pp. 2142-2144, Apr. 2011.

[28] R. Singh, A. K. Azad, Q. X. Jia, A. J. Taylor, and H. T. Chen, "Thermal tunability in terahertz metamaterials fabricated on strontium titanate single-crystal substrates," Opt. Lett., vol. 36, no. 7, pp. 1230-1232, Apr. 2011.

[29] S. Savo, D. Shrekenhamer, and W. J. Padilla, "Liquid crystal metamateria absorber spatial light modulator for THz applications," Adv. Opt. Mater. vol. 2, no. 3, pp. 275-279, Jan. 2014

[30] W. M. Zhu, A. Q. Liu, T. Bourouina, D. P. Tasi, J. H. Teng, X. H. Zhang, G. Q. Lo, D. L. Kwong, and N. I. Zheludev, "Microelectromechanical maltese-cross metamaterial with tunable terahertz anisotropy," Nature Commun., vol. 3, no. 1274, pp. 1-6, Dec. 2012.

[31] Y. H. Fu, A. Q. Liu, W. M. Zhu, X. M. Zhang, D. P. Tsai, J. B. Zhang, T. Mei, J. F. Tao, H. C. Guo, X. H. Zhang, J. H. Teng, N. I. Zheludev, G. Q. Lo, and D. L. Kwong, "A micromachined reconfigurable metamaterial via reconfiguration of asymmetric split-ring resonators," Adv. Funct. Mater. vol. 21, no. 18, pp. 3589-3594, Aug. 2011

[32] J. Y. Ou, E. Plum, J. Zhang, and N. I. Zheludev, "An electromechanically reconfigurable plasmonic metamaterial operating in the near-infrared," Nature Nanotechnol., vol. 8, no. 4, pp. 252-255, Mar. 2013.

[33] X. Liu and W. J. Padilla, "Dynamic manipulation of infrared radiation with MEMS metamaterials," Adv. Opt. Mater., vol. 1, no. 8, pp. 559-562, Jun. 2013.

[34] Y. S. Lin, Y. Qian, F. Ma, Z. Liu, P. Kropelnicki, and C. Lee, "Development of stress-induced curved actuators for a tunable $\mathrm{THz}$ filter based on double split-ring resonators," Appl. Phys. Lett., vol. 102, no. 11, p. 111908-1111908-5, Mar. 2013 
[35] Y. S. Lin, F. Ma, and C. Lee, "Three-dimensional movable metamaterial using electric split-ring resonators," Opt. Lett., vol. 38, no. 16, pp. 31263128, Aug. 2013.

[36] F. Ma, Y. Qian, Y. S. Lin, H. Liu, X. Zhang, Z. Liu, J. M.-L. Tsai, and C. Lee, "Polarization-sensitive microelectromechanical systems based tunable terahertz metamaterials using three dimensional electric split-ring resonator arrays," Appl. Phys. Lett., vol. 102, no. 16, p. 161912, Apr. 2013.

[37] P. Pitchappa, C. P. Ho, Y. S. Lin, P. Kropelnicki, C. Y. Huang, N. Singh, and C. Lee, "Micro-electro-mechanically tunable metamaterial with enhanced electro-optic performance," Appl. Phys. Lett., vol. 104, no. 15, p. 151104 , Apr. 2014.

[38] F. Ma, Y. S. Lin, X. Zhang, and C. Lee, "Tunable multiband terahertz metamaterials using a reconfigurable electric split-ring resonator array," Light, Sci. Appl., vol. 3, no. e171, pp. 1-8, May. 2014.

[39] C. P. Ho, P. Pitchappa, Y. S. Lin, C. Y. Huang, P. Kropelnicki, and C. Lee, "Electrothermally actuated microelectromechanical systems based omega-ring terahertz metamaterial with polarization dependent characteristics," Appl. Phys. Lett., vol. 104, no. 16, p. 161104, Apr. 2014.

[40] M. Shavezipur, W. Guo, C. Carraro, and R. Maboudian, "Characterization of adhesion force in MEMS at high temperature using thermally actuated microstructures," J. Microelectromech. Syst., vol. 21, no. 3, pp. 541-548, Jun. 2012.

[41] R. T. Chen, H. Nguyen, and M. C. Wu, "A high-speed low-voltage stressinduced micromachined $2 \times 2$ optical switch," IEEE Photon. Technol. Lett., vol. 11, no. 11, pp. 1396-1398, Nov. 1999.

Yu-Sheng Lin received the Ph.D. degree from the Institute of NanoEngineering and MicroSystems, National Tsing Hua University, Hsinchu, Taiwan, in 2011. He is currently a Research Fellow with the Department of Electrical and Computer Engineering, National University of Singapore, Singapore. His current research interests include metamaterial, nanofabrication techniques, and optical microelectromechanical systems.

Chia-Yi Huang received the Ph.D. degree from the Department of Photonics, National Cheng Kung University, Tainan, Taiwan, in 2010. He was employed as a Research Fellow at the Department of Electrical and Computer Engineering, National University of Singapore, Singapore. He is currently an Assistant Professor at Tunghai University, Taichung, Taiwan. His research interests include liquid crystal-based electro-optic devices, physics of metamaterials, and terahertz optics.
Chengkuo Lee (S'93-M'96) received the M.S. degree in materials science and engineering from the National Tsing Hua University, Hsinchu, Taiwan, in 1991, the M.S. degree in industrial and system engineering from Rutgers University, New Brunswick, NJ, USA, in 1993, and the Ph.D. degree in precision engineering from the University of Tokyo, Tokyo, Japan, in 1996.

He was a Foreign Researcher at the Nanometerscale Manufacturing Science Laboratory, Research Center for Advanced Science and Technology, University of Tokyo from 1993 to 1996 . He had also worked with the Techanical Engineering Laboratory, Advanced Industrial Science and Technology, Ministry of International Trade and Industry of Japan, as a Japan Science and Technology Research Fellow in 1996. Thereafter, he was a Senior Research Staff Member at the Microsystems Laboratory, Industrial Technology Research Institute, Hsinchu. In September 1997, he joined the Metrodyne Microsystem Corporation, Hsinchu, and established the Microelectromechanical Systems (MEMS) Device Division and the first micromachining laboratory for commercial purposes in Taiwan.

Dr. Lee was the Manager of the MEMS Device Division between 1997 and 2000. He was an Adjunct Assistant Professor at the Department of Electrophysics, National Chiao Tung University, Hsinchu, in 1998, and an Adjunct Assistant Professor at the Institute of Precision Engineering, National Chung Hsing University, Taichung, Taiwan, from 2001 to 2005. He cofounded Asia Pacific Microsystems, Inc. (APM), Hsinchu, in August 2001, and he became the Vice President of R\&D; then, later till the end of 2005, he became the Vice President of the optical communication business unit and the Special Assistant to the Chief Executive Officer in charge of international business and technica marketing for MEMS foundry service at APM, Inc., one of the top 30 MEMS manufacturers in the world in 2004. From 2006 to 2009, he was a Senior Member of Technical Staff at the Institute of Microelectronics, A*Star, Singapore. He is currently an Associate Professor at the Department of Electrical and Computer Engineering, National University of Singapore, Singapore. He is the coauthor of the book Advanced MEMS Packaging (McGraw-Hill, 2010). He has contributed more than 230 international conference papers and extended abstracts, 170 peer-reviewed international journal articles, and nine U.S. patents in the MEMS, NEMS, metamaterials, nanophotonics, and nanotechnology fields. 